\title{
A focus on dendritic cells and macrophages as key regulators of mucosal immunity
}

\section{BL Kelsall $^{1}$}

n $\mathrm{n}$ this issue, we have attempted to bring together recent findings on the ontogeny, phenotype, and function of dendritic cells (DCs) and macrophages at mucosal surfaces. Although both DCs and macrophages are members of the mononuclear phagocyte family, studies indicate that these cells are highly heterogeneous. Subpopulations have been defined that have specific localization and functional characteristics, yet we are early in our understanding of how these populations are developmentally or functionally related. To the end of gaining some clarity on these issues-or, more likely, of stimulating thought and discussion-we decided to publish a compilation of review articles from basic DC and macrophage biologists, along with immunologists with particular interests in mucosal tissues and the skin, to provide an additional perspective.

In the initial review, Priyanka Sathe and Ken Shortman discuss steady-state DC populations in the spleen and what is known regarding their development. They describe the major phenotypic differences in DC subpopulations, including plasmacytoid DCs (pDCs) and conventional DCs (cDCs), as well as the distinction between resident and migratory $\mathrm{cDCs}$, the latter of which come from DCs that capture self- or foreign antigens in peripheral tissues.
In addition, they address the cellular origin of these populations, indicating that $\mathrm{pDCs}$ arise in the bone marrow and circulate to lymphoid and nonlymphoid tissues, that resident $\mathrm{cDCs}$ are derived from a committed bone marrow-derived precursor population that differentiates into DCs within the local lymphoid tissues, and that migratory $\mathrm{cDCs}$ likely derive from blood monocytes. Furthermore, they address what is known (and not known) regarding the nature of progenitors for $\mathrm{cDCs}$, pDCs, and monocyte/macrophage lineages.

Next, David Hume presents his provocative view of the mononuclear phagocyte system (MPS). He provides evidence for common progenitors-but remarkable heterogeneity-within this system, which includes DCs, monocytes, and macrophages, and argues that many of our definitions of subpopulations of these cells are relatively arbitrary. In particular, he draws attention to commonly used markers, such as CD11c, langerin/CD207, CD11b, F4/80, CX3CR1, and CD68/ macrosialin in the mouse, and indicates that it is not clear that any one or a combination of these (or other) markers is sufficient to distinguish "DCs" from "macrophages." While there are functional subpopulations of cells within specific organs, or locations within organs that are enriched for particular surface markers, 
there are also markers and receptors that are heterogeneously expressed on individual cells, which Hume suggests is due to a stochastic process of gene expression. The end result is an MPS of immense functional diversity that may be important for responding to the complexity of microbial pathogens.

The remaining articles address recent findings in the origin, phenotype, and function of DCs and macrophages in specific tissues, including the lung (by Corine GeurtsvanKessel and Bart Lambrecht), the genitourinary tract (by Norifumi Iijima, Joseph Thompson, and Akiko Iwasaki), and the intestine (by me) - and finally, the skin (by Mark Udey and Keisuke Nagao). Although all the reviews highlight the diversity of cell types, locations, and functional attributes of DC and macrophage populations, several obvious common aspects of these systems have emerged. The first is the distinction between lymph node-resident and migrating $\mathrm{CDC}$ populations which may develop from distinct progenitor populations and have unique functions. In particular, migrating DCs in the steady state appear to induce appropriate immune responses for the maintenance of tissue and immunological homeostasisincluding the induction of Tregs and noninflammatory or homeostatic T- and $\mathrm{B}$-cell responses. Furthermore, there is likely a primary influence of the tissue microenvironment in the conditioning of migrating DC populations, which includes factors such as transforming growth factor- $\beta$ and IL-10.
Second, tissue macrophages in the steady state appear to have a predominant role in innate immunity, including the ability to phagocytose and kill bacteria, produce immunoregulatory cytokines, and provide signals for tissue remodeling and repair. Whether tissue macrophages present antigens to naive $\mathrm{T}$ cells or have additional roles in the maintenance or expansion of T-cell populations within peripheral tissues is not yet clear. Third, infection or tissue inflammation fundamentally changes the dynamics, the developmental pathways, and the function of DC and macrophage populations. Common features are the recruitment of populations of monocytes that differentiate into inflammatory rather than suppressive or regulatory cells, and the increased turnover and migration of $\mathrm{cDCs}$ to draining lymphoid tissues. Moreover, there is increasing evidence that $\mathrm{pDCs}$ are recruited to inflamed or infected tissues, where they may contribute to innate defense or local inflammation.

The goal in putting together this issue was to present new information from studies of multiple related organ systems, along with basic reviews of DC and macrophage development. We hope this issue of Mucosal Immunology will be a valuable stimulus for thought and future research, and will help us all develop a broader conceptual framework for understanding the complexities of the MPS in mucosal tissues.

C 2008 Society for Mucosal Immunology 